\title{
Bulk-boundary correspondence in a non-Hermitian system in one dimension with chiral inversion symmetry
}

\author{
L. Jin* and Z. Song \\ School of Physics, Nankai University, Tianjin 300071, China
}

(Received 9 September 2018; revised manuscript received 10 January 2019; published 1 February 2019)

\begin{abstract}
Asymmetric coupling amplitudes effectively create an imaginary gauge field, which induces a non-Hermitian Aharonov-Bohm (AB) effect. Nonzero imaginary magnetic flux invalidates the bulk-boundary correspondence and leads to a topological phase transition. However, the way of non-Hermiticity appearance may alter the system topology. By alternatively introducing the non-Hermiticity under symmetry to prevent nonzero imaginary magnetic flux, the bulk-boundary correspondence recovers and every bulk state becomes extended; the bulk topology of the Bloch Hamiltonian predicts the (non)existence of edge states and topological phase transition. These are elucidated in a non-Hermitian Su-Schrieffer-Heeger model, where chiral inversion symmetry ensures the vanishing of imaginary magnetic flux. The average value of Pauli matrices under the eigenstate of chiralinversion-symmetric Bloch Hamiltonian defines a vector field; the vorticity of topological defects in the vector field is a topological invariant. Our findings are applicable in other non-Hermitian systems. We first uncover the roles played by the non-Hermitian $\mathrm{AB}$ effect and chiral inversion symmetry for the breakdown and recovery of bulk-boundary correspondence, and develop new insights for understanding the non-Hermitian topological phases of matter.
\end{abstract}

DOI: 10.1103/PhysRevB.99.081103

Introduction. Topological theory has been well established in condensed-matter physics [1-32] and recent experimental progress in optics boosts the development of topological photonics [33-41]. The existence of gapless edge states of a system under the open boundary condition (OBC) is predictable from the change of topological invariants associated with the bulk topology of the system under the periodical boundary condition (PBC), known as the (conventional) bulkboundary correspondence, which is ubiquitously applicable in Hermitian systems.

In parallel, non-Hermitian physics exhibits considerable intriguing features [42-75]; the unexpected novel interface states appear between non-Hermitian periodic media with distinct topologies [76-89]. These stimulate the studies of topological phases and edge states in non-Hermitian systems [90-117]. Non-Hermitian band theory and the topological characterization are developed employing the left and right eigenstates [48,52]; the Chern number, generalized Berry phase, and winding numbers are quantized as topological invariants [107-109].

Remarkably, the bulk-boundary correspondence [118] is invalid in certain non-Hermitian topological systems [119-121]: Systems under PBC and OBC have dramatically different energy spectra, and all the eigenstates local-

\footnotetext{
*jinliang@ nankai.edu.cn
}

Published by the American Physical Society under the terms of the Creative Commons Attribution 4.0 International license. Further distribution of this work must maintain attribution to the author(s) and the published article's title, journal citation, and DOI. ize near system boundaries (the non-Hermitian skin effect) [122-124]. These have received great research interests in non-Hermitian systems of the asymmetric Su-SchriefferHeeger (SSH) model, topological insulators, and nodal-line semimetals [122-131]. Biorthogonal [122] and non-Bloch bulk-boundary correspondences [123] are suggested. In contrast, non-Hermiticity does not inevitably destroy the bulkboundary correspondence [77,78,93-97], which is verified in a parity-time-symmetric non-Hermitian SSH model with staggered couplings and losses [85-92]. Questions arise: Why does bulk-boundary correspondence fail in certain nonHermitian systems? What roles do non-Hermiticity and symmetry play in the breakdown of bulk-boundary correspondence? How to characterize the topological properties and understand the topological invariant without (conventional) bulk-boundary correspondence?

In this Rapid Communication, we first report that chiralinversion symmetry plays an important role for the bulkboundary correspondence in the non-Hermitian system of a non-Hermitian SSH model with asymmetric coupling, which leads to a non-Hermitian Aharonov-Bohm (AB) effect with an imaginary magnetic flux under PBC and a non-Hermitian skin effect under $\mathrm{OBC}$ without chiral inversion symmetry. The imaginary magnetic flux results in a complex spectrum and a topological phase transition, but it disappears under $\mathrm{OBC}$; the OBC spectrum significantly differs from the $\mathrm{PBC}$ spectrum, and the bulk-boundary correspondence fails. The non-Hermitian $\mathrm{AB}$ effect vanishes if the asymmetry is alternatively introduced without breaking chiral inversion symmetry. The bulk-boundary correspondence is valid; a topological invariant is constructed from the system bulk with the imaginary gauge field removed, being the vorticity of band touching 


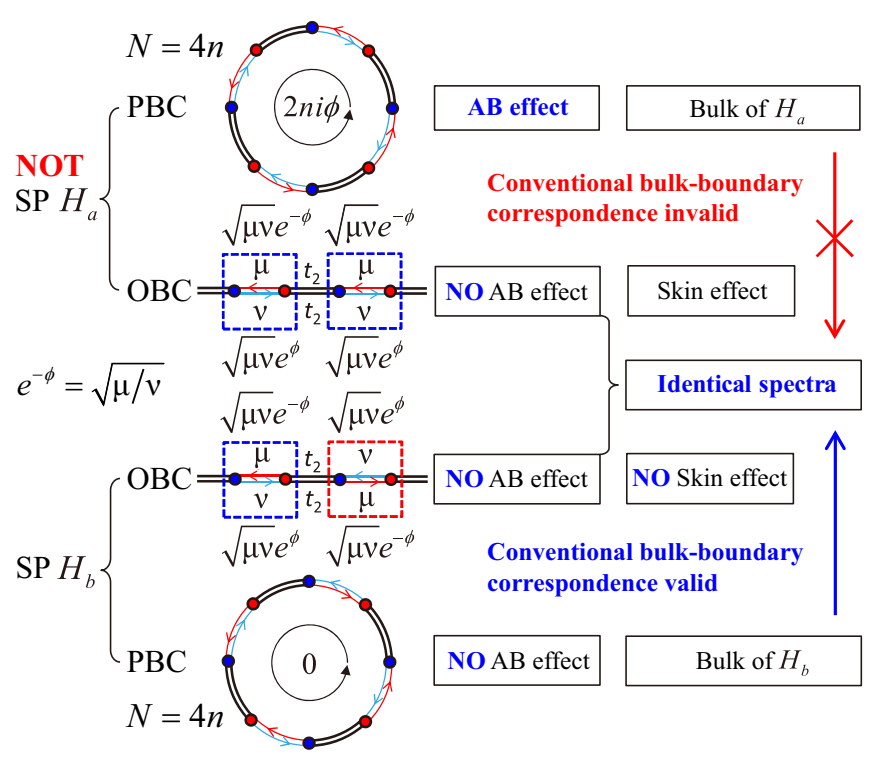

FIG. 1. Breakdown (recovery) of bulk-boundary correspondence for the system without (with) chiral inversion symmetry from the viewpoint of the non-Hermitian $\mathrm{AB}$ effect. Imaginary gauge field induces nonzero (zero) imaginary magnetic flux in $H_{a}\left(H_{b}\right)$ under PBC. Topological invariant obtained from the bulk Bloch Hamiltonian of system $b$ is a Bloch (non-Bloch) topological invariant for system $b$ (a). Lattice size is $N=4 n$.

points as topological defects in the vector field defined from the average values of Pauli matrices. Our findings are valid for other non-Hermitian topological systems.

Topological phase transition induced by symmetry breaking. The Bloch Hamiltonian of a non-Hermitian system $a$ (Fig. 1) under PBC is

$$
H_{a}(k)=\left(t_{1}+t_{2} \cos k\right) \sigma_{x}+\left(t_{2} \sin k-i \gamma\right) \sigma_{y}
$$

where $\sigma_{x, y}$ are the Pauli matrices. $t_{2}$ is the intercell coupling. Set $\mu=t_{1}-\gamma$ and $v=t_{1}+\gamma$; the asymmetric intracell coupling amplitude $\left(\mu \neq v^{*}\right)$ raises the non-Hermiticity. Non-Hermitian asymmetric coupling can be realized between primary resonators evanescently coupled through an auxiliary resonator [132-134], which has half perimeter gain and half perimeter loss, leading to the amplification and attenuation for the coupling amplitudes in opposite tunneling directions. Implementation of asymmetric coupling with ultracold atoms in an optical lattice is possible [124].

In the Hermitian case $(\gamma=0)$, system $a$ holds chiralinversion symmetry

$$
(\mathcal{S P}) H_{a}(k)(\mathcal{S P})^{-1}=-H_{a}(-k), \quad U_{\mathcal{S P}} H_{a} U_{\mathcal{S P}}^{-1}=-H_{a} .
$$

The constraints are for a combined chiral inversion symmetry. $\mathcal{S P}$ and $U_{\mathcal{S P}}$ are unitary operators. $H_{a}\left[H_{a}(k)\right]$ is the Hamiltonian in the real space ( $k$ space). Two band touching degeneracy points exist [Fig. 2(d)].

In the non-Hermitian case $(\gamma \neq 0)$, unlike the alternative gain and loss [86-92], the asymmetric coupling breaks the chiral inversion symmetry $\left(H_{a}\right.$ in Fig. 1$)$. Taking $\mu \nu>0$ as an
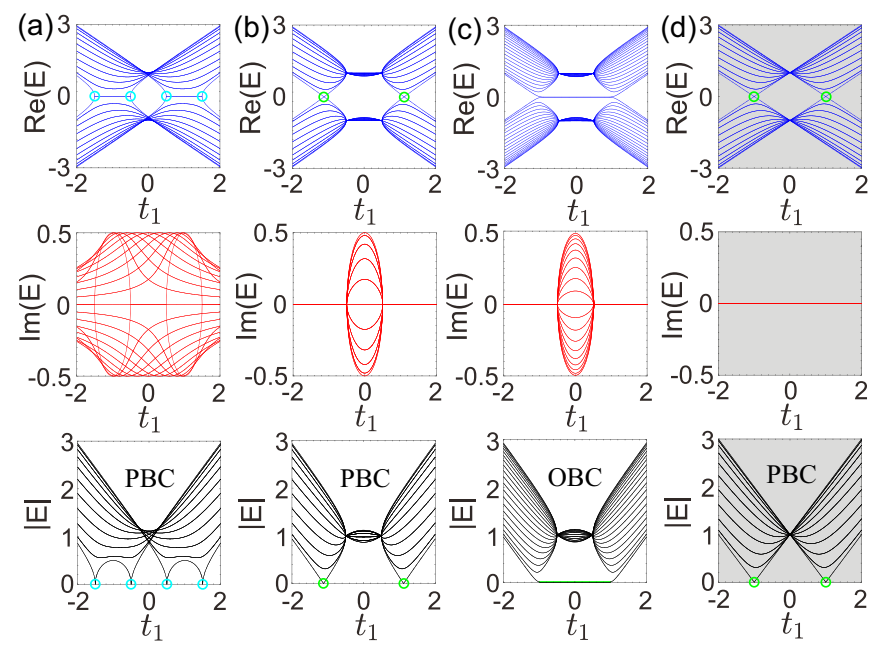

FIG. 2. (a) [(b)] Energy spectrum for $H_{a}\left(H_{b}\right)$ under PBC. (c) Identical spectra of $H_{a}$ and $H_{b}$ under OBC with one intercell coupling $t_{2}$ missing, and (d) $H_{a}$ and $H_{b}$ under PBC in the Hermitian case $(\gamma=0)$. The band touching exceptional points (degeneracy points) are indicated by the cyan (green) hollow circles. The system parameters are $N=40, t_{2}=1$, and $\gamma=1 / 2$ in (a)-(c).

illustration (see Supplemental Material A [135]) and rewritten

$$
\mu=\sqrt{\mu \nu} e^{-\phi}, \quad v=\sqrt{\mu \nu} e^{\phi},
$$

where $e^{-\phi} \equiv \sqrt{\mu / \nu}[133,134,136]$, the asymmetric coupling is expressed as a symmetric coupling $\sqrt{\mu \nu}$ with Peierls "phase" factor [37-41,137-143] of amplification/attenuation $e^{ \pm \phi}$ [144], which indicates the presence of an imaginary gauge field [132-134]. A non-Hermitian AB phase factor of amplification/attenuation $e^{ \pm i(2 n i \phi)}$ is accumulated when a particle is circling a loop in $H_{a}$ under PBC, where 2ni $\phi$ is the imaginary magnetic flux [140-144]. The eigenvalues are

$$
E_{a, \pm}= \pm \sqrt{t_{2}^{2}+\mu \nu+2 t_{2} \sqrt{\mu \nu} \cos (k+i \phi)}
$$

with $k=\pi m / n$, integer $m \in[1,2 n]$ [Fig. 2(a)].

In contrast to a real magnetic flux that shifts $k$ in the momentum space without varying the dispersion relation [140], the momentum changes to $k+i \phi[125,126,129]$ and the spectrum becomes fully complex affected by imaginary magnetic flux, which induces a topological phase transition with band touching degeneracy points split into pairs of band touching exceptional points (EPs) [Figs. 2(a) and 2(d)] [71] that exhibit different topology [145-161]. Imaginary magnetic flux is absent under OBC, thus the spectra and band touching points under PBC [Fig. 2(a)] and OBC [Fig. 2(c)] are dramatically different [119-128]. The eigenstate amplitude is one-way enlarged under OBC because of an imaginary gauge field [133,134,162]; and all the eigenstates localize at the system boundary (non-Hermitian skin effect [123-127]). The localization length is $\xi=\phi^{-1}$ [163]. The inverse participation ratio (IPR) $\sum_{j}\left|\psi_{j}\right|^{4} /\left(\sum_{j}\left|\psi_{j}\right|^{2}\right)^{2}$ of bulk states scales as $N^{-1}$ for small $N$, particularly for weak non-Hermiticity; and becomes system size insensitive when the localization dominates at large $N$ (see Supplemental Material B [135]).

Bulk-boundary correspondence. Chiral inversion symmetry holds when non-Hermiticity is alternatively introduced in 
system $b$ ( $H_{b}$ in Fig. 1) [164]. Under symmetry protection, two degeneracy points move without splitting into EP pairs [Figs. 2(b) and 2(d)]. The eigenstates under OBC are symmetric/antisymmetric. All bulk states are extended and the non-Hermitian skin effect disappears even though most bulk states have complex eigenvalues (the IPR of the bulk states of system $b$ is inversely proportional to the system size [135]). Although the significant difference between eigenstates, systems $a$ and $b$, under OBC possess identical energy spectra (see Supplemental Material C [135]), the imaginary gauge fields do not affect OBC spectra [Fig. 2(c)]. These indicate that the way of non-Hermiticity appearance affects system topology. In particular, the non-Hermiticity solely induces nontrivial topology at $t_{1}=t_{2}$ [165].

The amplification and attenuation cancel in $H_{b}$. The combined chiral inversion $(\mathcal{S P})$ symmetry prevents the appearance of nonzero imaginary magnetic flux and the bulkboundary correspondence is valid [Figs. 2(b) and 2(c)] (also in Refs. [77,78,86-94], but is invalid in Refs. [119-130] without chiral inversion symmetry), while individual chiral and inversion symmetries do not necessarily hold separately (see Supplemental Material D [135]). The (non)existence of topologically protected edge states is predictable from the bulk of system $b$,

$$
H_{b}(k)=\left(\begin{array}{cccc}
0 & \sqrt{\mu \nu} e^{-\phi} & 0 & t_{2} e^{-i k} \\
\sqrt{\mu \nu} e^{\phi} & 0 & t_{2} & 0 \\
0 & t_{2} & 0 & \sqrt{\mu \nu} e^{\phi} \\
t_{2} e^{i k} & 0 & \sqrt{\mu \nu} e^{-\phi} & 0
\end{array}\right) .
$$

Through a similar transformation with only nonzero diagonal elements $U_{\mu \nu}=\operatorname{diag}(\sqrt{\nu}, \sqrt{\mu}, \sqrt{\mu}, \sqrt{v})$, the imaginary gauge fields (factors $e^{ \pm \phi}$ ) are removed from $H_{b}$ (see Supplemental Material E [135]); and we obtain $U_{\mu \nu} H_{b}(k) U_{\mu \nu}^{-1}$, which is equivalent to a two-site unit cell bulk $h_{b}(k)=(\sqrt{\mu \nu}+$ $\left.t_{2} \cos k\right) \sigma_{x}+\left(t_{2} \sin k\right) \sigma_{y}$. The eigenvalues are

$$
E_{b, \pm}= \pm \sqrt{t_{2}^{2}+\mu \nu+2 t_{2} \sqrt{\mu \nu} \cos (k)},
$$

where $k=\pi m / n, m \in[1,2 n]$. The bulk topology of $h_{b}(k)$ correctly predicts the (non)existence of edge states in both systems $a$ and $b$ under OBC (Fig. 1) [135]. Removing the imaginary gauge field in system bulk gives $h_{b}(k)$, which is identical with that found by solving the open system [123].

For $\gamma=|r| e^{i \theta}(-\pi \leqslant \theta \leqslant \pi)$, the band gap closes at

$$
\left(t_{1}^{2}-|r|^{2}\right)^{2}+4 t_{1}^{2}|r|^{2} \sin ^{2} \theta=t_{2}^{4},
$$

and $\cos ^{2}(k)=\left[t_{2}^{2}+t_{1}^{2}-|r|^{2} \cos (2 \theta)\right] /\left(2 t_{2}^{2}\right)$. The finite size effects appear in discrete systems (see Supplemental Material F [135]). For real $\mu$ and $\nu$ at $\theta=0$, the band touching points are degeneracy (exceptional) points at $t_{1}^{2}=+(-) t_{2}^{2}+$ $\gamma^{2}[122,123]$, being topological defects carrying integer (halfinteger) vorticity. The band touching EPs only appear for $\gamma^{2}>t_{2}^{2}$.

Topological invariant. Topology invariants are recently constructed in non-Hermitian systems [94,107-109,112,123]. The Chern number defined via Berry curvature $[94,108]$, the vorticity defined via the complex energy [109], and the
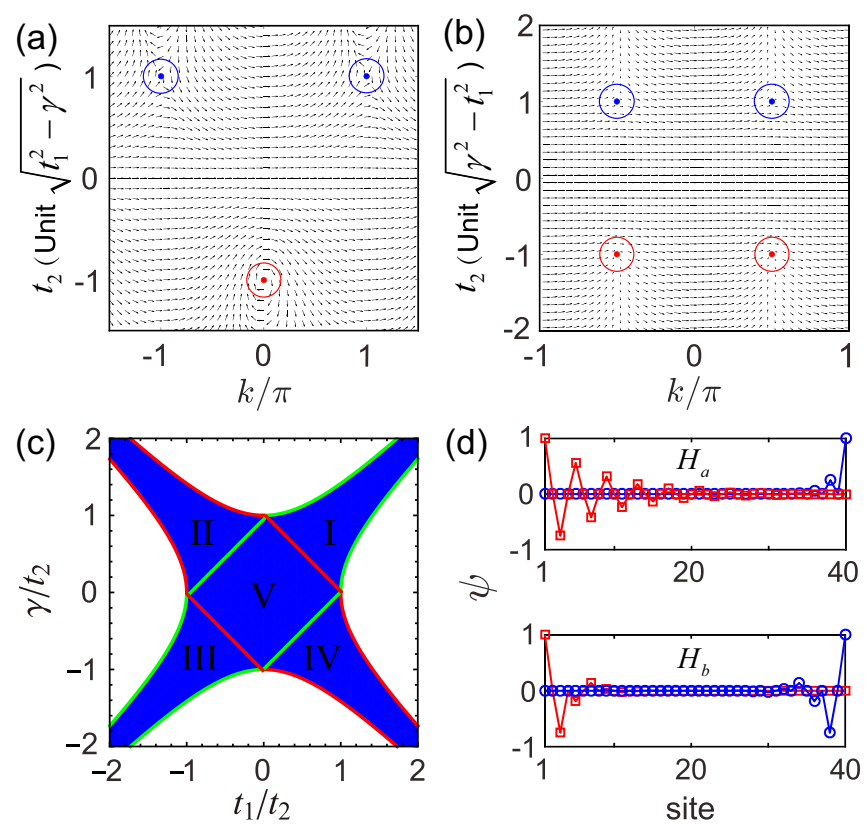

FIG. 3. Vector field $\mathbf{F}(\mathbf{k})=\left(\left\langle\sigma_{x}\right\rangle,\left\langle\sigma_{y}\right\rangle\right)$ associated with $E_{b,+}$ of $h_{b}(k)$ for (a) $\mu \nu=t_{1}^{2}-\gamma^{2}>0$ and (b) $\mu \nu=t_{1}^{2}-\gamma^{2}<0$. Red (blue) circles indicate the topological defects with vortices (antivortices), which appear at $\left(k, t_{2}\right)=\left(0,-\sqrt{t_{1}^{2}-\gamma^{2}}\right)$ or $\left( \pm \pi, \sqrt{t_{1}^{2}-\gamma^{2}}\right)$ in (a) and at $\left(k, t_{2}\right)=\left( \pm \pi / 2, \pm \sqrt{\gamma^{2}-t_{1}^{2}}\right)$ in (b). (c) Phase diagram for real $\gamma$. Two topological zero edge states exist in the blue region $-t_{2}^{2}<\mu \nu<t_{2}^{2}$ for one intercell coupling $t_{2}$ missing. (d) Zero edge states for systems $a$ and $b$ under OBC. The system parameters are $N=40, t_{1}=1 / 4, \gamma=1 / 2$, and $t_{2}=1$.

generalized Berry phase defined via the argument of effective magnetic field $[107,108,112]$ are quantized. The vorticity of topological defects in a vector field $\mathbf{B}(\mathbf{k})$ associated with the Bloch Hamiltonian is a topological invariant [166,167]; we generalize this vorticity to non-Hermitian systems through defining a two-component vector field $\mathbf{F}(\mathbf{k})=\left(\left\langle\sigma_{x}\right\rangle,\left\langle\sigma_{y}\right\rangle\right)$ [Figs. 3(a) and 3(b)] that is composed of the average values of Pauli matrices under the eigenstates of $h_{b}(k) . w=$ $\oint_{L}(2 \pi)^{-1}\left(\hat{F}_{x} \nabla \hat{F}_{y}-\hat{F}_{y} \nabla \hat{F}_{x}\right) \mathrm{d} \mathbf{k}$ characterizes the vorticity of topological defects inside the loop $L$ in the parameter plane $\mathbf{k}=\left(k, t_{2}\right)$, where $\hat{F}_{x(y)}=F_{x(y)} / \sqrt{F_{x}^{2}+F_{y}^{2}}$ and $\nabla=\partial / \partial \mathbf{k}$, which is in accord with that defined in the Brillouin zone of a two-dimensional (2D) brick wall lattice (see Supplemental Material E [135]). The varying direction of $\mathbf{F}(\mathbf{k})$ accumulated is $2 \pi w= \pm 2 \pi( \pm \pi)$ in Fig. 3(a) [Fig. 3(b)] if $L$ encircles a topological defect, the plus (minus) sign corresponds to the vortex (antivortex); otherwise, if $L$ does not encircle a topological defect, the varying direction is $2 \pi w=0$.

The phase diagram is plotted in Fig. 3(c) for real $\gamma$. For $\mu \nu>0$, the degeneracy points are at $t_{2}^{2}-\mu \nu=0$. As nonHermiticity increases, the band gap inside two EPs $[48,74,75]$ with complex spectrum diminishes and closes at $t_{1}=0$ when $\gamma^{2}=t_{2}^{2} \cdot \mu \nu=0\left(t_{1}= \pm \gamma\right)$ are EPs, where the eigenstates are highly defective and fully constituted by two-state coalescences at energy $\pm t_{2}$. For $\mu \nu<0, t_{2}^{2}+\mu \nu=0$ yields another boundary for the zero edge states determined from band touching EPs. Two topological zero edge states exist in 
(a)

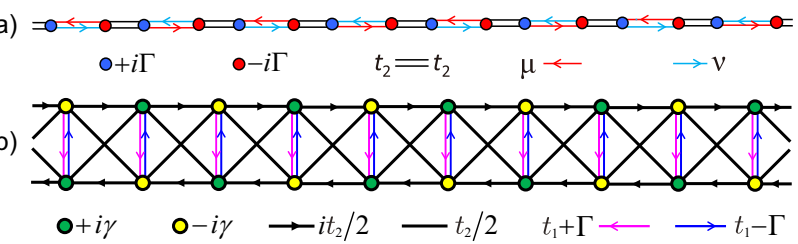

FIG. 4. Chiral inversion symmetric (a) non-Hermitian SSH model of system $b$ in Fig. $1\left(H_{b}\right)$ with staggered gain and loss $\Gamma$ and (b) non-Hermitian Creutz ladder that is equivalent to (a).

the regions $\gamma^{2}-t_{2}^{2}<t_{1}^{2}<\gamma^{2}+t_{2}^{2}$ for one intercell coupling $t_{2}$ vanishing under OBC [168].

Topological edge states. The bulk topology relates to the (dis)appearance of edge states at the interfaces where the topological invariant $(w)$ changes. We consider systems with complete unit cells $(N=4 n)$ in which one $t_{2}$ vanishes (see Supplemental Material G for the case with a defective unit cell [135]). In system $b$, two edge states localize on the left and right boundaries, respectively, in all blue regions of Fig. 3(c). In system $a$, this occurs only in region V; and both edge states localize on the right (left) boundary in regions I and III (II and IV).

For system $b$, the left edge state is $\psi_{2 j}=0$ and

$$
\psi_{2 j+1}=-\left[(\mu+v)+(-1)^{j}(\mu-v)\right] /\left(2 t_{2}\right) \psi_{2 j-1},
$$

at $N \gg 1$. The right edge state is a left-right spatial reflection of the left edge state [Fig. 3(d)]. Anomalous edge states localize in one unit cell at system boundary at the EPs $\left(t_{1}^{2}=\right.$ $\gamma^{2}$ ) $[107,119,120]$. At $t_{1}=-\gamma$, the left (right) edge state is $\psi_{1}=1\left(\psi_{N}=1\right)$; at $t_{1}=\gamma$, the left edge state is $\psi_{1}=$ $-(+) \psi_{3}=1$ and the right edge state is $\psi_{N}=-(+) \psi_{N-2}=1$ when $t_{1} / t_{2}>0\left(t_{1} / t_{2}<0\right)$.

In contrast, for system $a$, the left edge state is $\psi_{2 j}=0$ and $\psi_{2 j+1}=\left(-v / t_{2}\right) \psi_{2 j-1}$; the right edge state is $\psi_{2 j-1}=0$ and $\psi_{N-2 j}=\left(-\mu / t_{2}\right) \psi_{N+2-2 j}$ with a different decay rate $-\mu / t_{2}$ [Fig. 3(d)]. The imaginary gauge field induces imbalanced probability distributions between edge states. The green (red) ribbon in Fig. 3(c) indicates $\left|\mu / t_{2}\right|<1\left(\left|v / t_{2}\right|<1\right)$; both edge states localize on the right (left) boundary. The edge states are $\psi_{1}=1\left(\psi_{N}=1\right)$ for $t_{1} / t_{2}<0\left(t_{1} / t_{2}>0\right)$ at the EPs.

Discussion and conclusion. Figure 4(a) depicts the chiralinversion-symmetric non-Hermitian SSH model of system $b$ with staggered gain and loss $\Gamma$, where chiral symmetry and inversion symmetry are not separately held. The system shown in Fig. 4(a) is equivalent to the chiral inversion symmetric non-Hermitian Creutz ladder [Fig. 4(b)]. The Creutz ladder has a $\pi$ magnetic flux in each plaquette [169]. The Creutz ladder in Refs. [119-121] is equivalent to system $a$ through a similar transformation $U=I_{2 n} \otimes\left(i \sigma_{x}+I_{2}\right)$ (see Supplemental Material H [135]), where bulk-boundary correspondence fails because the gain and loss associated with real magnetic flux breaks chiral inversion symmetry and effectively creates imaginary magnetic flux under PBC.

Time-reversal (inversion) symmetry prevents nonzero real (imaginary) magnetic flux. An attenuation (amplification) factor $e^{-\phi}$ accompanied with the corresponding amplification (attenuation) factor $e^{\phi}$ in the direction concerned can prevent nonzero imaginary magnetic flux. This is enabled under inversion symmetry $(\mathcal{P})$ or combined inversion symmetries such as chiral inversion $(\mathcal{S P})$ symmetry, charge-conjugation inversion $(\mathcal{C P})$ symmetry, and parity-time $(\mathcal{P} \mathcal{T})$ symmetry. For a $2 \mathrm{D}$ non-Hermitian Chern insulator $\left(m+t \cos k_{x}+t \cos k_{y}\right) \sigma_{x}+\left(t \sin k_{x}+\right.$ $i \gamma) \sigma_{y}+t \sin k_{y} \sigma_{z} \quad$ [130], we write its energy bands as $\pm \sqrt{\mu \nu+t^{2}+t^{2} \sin ^{2} k_{y}+2 t \sqrt{\mu \nu} \cos \left(k_{x}+i \phi\right)}$, where we set $\mu=m+t \cos k_{y}+\gamma, v=m+t \cos k_{y}-\gamma$, and $\sqrt{\mu / v}=$ $e^{-\phi}$ (for $\mu \nu>0$ ). An imaginary magnetic flux exists in the $x$ direction, but not in the $y$ direction; a considerable difference between PBC and OBC spectra is observed in the $x$ direction [130]. Introducing the non-Hermiticity under inversion symmetry prevents nonzero imaginary magnetic flux under PBC because of the cancellation between amplification and attenuation factors $e^{ \pm \phi}$ in the $x$ direction and enables the bulk-boundary correspondence. By applying the same procedure done for the non-Hermitian SSH model of system $b$, we can obtain an equivalent bulk Bloch Hamiltonian $h_{b, C I}\left(k_{x}, k_{y}\right)=\left(\sqrt{\mu \nu}+t \cos k_{x}\right) \sigma_{x}+$ $\left(t \sin k_{x}\right) \sigma_{y}+\left(t \sin k_{y}\right) \sigma_{z}$ after removing the imaginary gauge field (see Supplemental Material I [135]). The energy bands are $\pm \sqrt{\mu \nu+t^{2}+t^{2} \sin ^{2} k_{y}+2 t \sqrt{\mu \nu} \cos k_{x}}$. The bulk topology of $h_{b, C I}\left(k_{x}, k_{y}\right)$ correctly predicts the topological phase transition and the (non)existence of edge states for the Chern insulator under OBC.

Bulk-boundary correspondence fails for nonzero imaginary magnetic flux under PBC if the flux vanishes under OBC; the bulk-boundary correspondence recovers by alternatively introducing non-Hermiticity under symmetry, which prevents nonzero imaginary magnetic flux; and a topological invariant can be constructed from the bulk Bloch Hamiltonian. The non-Bloch topological invariant and exotic bulk-boundary correspondence $[123,124]$ are elaborated from the viewpoint of (conventional) bulk-boundary correspondence. Our findings provide new insights from non-Hermitian $\mathrm{AB}$ effect and shed light on non-Hermitian topological phases of matter.

Acknowledgments. We acknowledge the support of NSFC (Grants No. 11605094 and No. 11874225).
[1] A. Y. Kitaev, Unpaired Majorana fermions in quantum wires, Phys. Usp. 44, 131 (2001).

[2] S. Ryu and Y. Hatsugai, Topological Origin of Zero-Energy Edge States in Particle-Hole Symmetric Systems, Phys. Rev. Lett. 89, 077002 (2002).

[3] M. Greiner, O. Mandel, T. Esslinger, T. W. Hänsch, and I. Bloch, Quantum phase transition from a superfluid to a Mott insulator in a gas of ultracold atoms, Nature (London) 415, 39 (2002).

[4] S. Murakami, N. Nagaosa, and S.-C. Zhang, Spin-Hall Insulator, Phys. Rev. Lett. 93, 156804 (2004).

[5] C. L. Kane and E. J. Mele, Quantum Spin Hall Effect in Graphene, Phys. Rev. Lett. 95, 226801 (2005). 
[6] B. A. Bernevig, T. L. Hughes, and S. C. Zhang, Quantum spin Hall effect and topological phase transition in HgTe quantum wells, Science 314, 1757 (2006).

[7] L. Fu and C. L. Kane, Topological insulators with inversion symmetry, Phys. Rev. B 76, 045302 (2007).

[8] L. Fu, C. Kane, and E. Mele, Topological Insulators in Three Dimensions, Phys. Rev. Lett. 98, 106803 (2007).

[9] A. P. Schnyder, S. Ryu, A. Furusaki, and A. W. W. Ludwig, Classification of topological insulators and superconductors in three spatial dimensions, Phys. Rev. B 78, 195125 (2008).

[10] H. Zhang, C.-X. Liu, X.-L. Qi, X. Dai, Z. Fang, and S.C. Zhang, Topological Insulators in the $\mathrm{Bi}_{2} \mathrm{Se}_{3}, \mathrm{Bi}_{2} \mathrm{Te}_{3}$ and $\mathrm{Sb}_{2} \mathrm{Te}_{3}$ with a single Dirac cone on the surface, Nat. Phys. 5, 438 (2009).

[11] S. Ryu, A. P. Schnyder, A. Furusaki, and A. W. W. Ludwig, Topological insulators and superconductors: Tenfold way and dimensional hierarchy, New J. Phys. 12, 065010 (2010).

[12] M. Z. Hasan and C. L. Kane, Colloquium: Topological insulators, Rev. Mod. Phys. 82, 3045 (2010).

[13] G. Xu, H. Weng, Z. Wang, X. Dai, and Z. Fang, Chern Semimetal and the Quantized Anomalous Hall Effect in $\mathrm{HgCr}_{2} \mathrm{Se}_{4}$, Phys. Rev. Lett. 107, 186806 (2011).

[14] A. A. Burkov and L. Balents, Weyl Semimetal in a Topological Insulator Multilayer, Phys. Rev. Lett. 107, 127205 (2011).

[15] X.-L. Qi and S.-C. Zhang, Topological insulators and superconductors, Rev. Mod. Phys. 83, 1057 (2011).

[16] S. M. Young, S. Zaheer, J. C. Y. Teo, C. L. Kane, E. J. Mele, and A. M. Rappe, Dirac Semimetal in Three Dimensions, Phys. Rev. Lett. 108, 140405 (2012).

[17] Z. Wang, Y. Sun, X.-Q. Chen, C. Franchini, G. Xu, H. Weng, $\mathrm{X}$. Dai, and Z. Fang, Dirac semimetal and topological phase transitions in $A_{3} \mathrm{Bi}(A=\mathrm{Na}, \mathrm{K}, \mathrm{Rb})$, Phys. Rev. B 85, 195320 (2012).

[18] C.-E. Bardyn, M. A. Baranov, E. Rico, A. İmamoğlu, P. Zoller, and S. Diehl, Majorana Modes in Driven-Dissipative Atomic Superfluids with a Zero Chern Number, Phys. Rev. Lett. 109, 130402 (2012).

[19] L. Tarruell, D. Greif, T. Uehlinger, G. Jotzu, and T. Esslinger, Creating, moving and merging Dirac points with a Fermi gas in a tunable honeycomb lattice, Nature (London) 483, 302 (2012).

[20] Z. Liu, D. L. Kovrizhin, and E. J. Bergholtz, Bulk-edge correspondence in fractional Chern insulators, Phys. Rev. B 88, 081106(R) (2013).

[21] Z. Wang, H. Weng, Q. Wu, X. Dai, and Z. Fang, Threedimensional Dirac semimetal and quantum transport in $\mathrm{Cd}_{3} \mathrm{As}_{2}$, Phys. Rev. B 88, 125427 (2013).

[22] X.-J. Liu, K. T. Law, T. K. Ng, and P. A. Lee, Detecting Topological Phases in Cold Atoms, Phys. Rev. Lett. 111, 120402 (2013).

[23] D.-L. Deng, S.-T. Wang, and L.-M. Duan, Direct probe of topological order for cold atoms, Phys. Rev. A 90, 041601(R) (2014).

[24] Z. K. Liu, B. Zhou, Y. Zhang, Z. J. Wang, H. M. Weng, D. Prabhakaran, S.-K. Mo, Z. X. Shen, Z. Fang, X. Dai, Z. Hussain, and Y. L. Chen, Discovery of a three-dimensional topological Dirac semimetal, $\mathrm{Na}_{3} \mathrm{Bi}$, Science 343, 864 (2014).

[25] M. Xiao, G. Ma, Z. Yang, P. Sheng, Z. Q. Zhang, and C. T. Chan, Geometric phase and band inversion in periodic acoustic systems, Nat. Phys. 11, 240 (2015).
[26] H. Weng, C. Fang, Z. Fang, B. A. Bernevig, and X. Dai, Weyl Semimetal Phase in Noncentrosymmetric Transition-Metal Monophosphides, Phys. Rev. X 5, 011029 (2015).

[27] L. Lu, Z. Wang, D. Ye, L. Ran, L. Fu, J. D. Joannopoulos, and M. Soljačić, Experimental observation of Weyl points, Science 349, 622 (2015).

[28] D. Leykam, M. C. Rechtsman, and Y. D. Chong, Anomalous Topological Phases and Unpaired Dirac Cones in Photonic Floquet Topological Insulators, Phys. Rev. Lett. 117, 013902 (2016).

[29] C.-K. Chiu, J. C. Y. Teo, A. P. Schnyder, and S. Ryu, Classification of topological quantum matter with symmetries, Rev. Mod. Phys. 88, 035005 (2016).

[30] F. K. Kunst, M. Trescher, and E. J. Bergholtz, Anatomy of topological surface states: Exact solutions from destructive interference on frustrated lattices, Phys. Rev. B 96, 085443 (2017).

[31] F. K. Kunst, G. van Miert, and E. J. Bergholtz, Lattice models with exactly solvable topological hinge and corner states, Phys. Rev. B 97, 241405 (2018).

[32] N. P. Armitage, E. J. Mele, and A. Vishwanath, Weyl and Dirac semimetals in three-dimensional solids, Rev. Mod. Phys. 90, 015001 (2018).

[33] M. Atala, M. Aidelsburger, J. T. Barreiro, D. Abanin, Takuya Kitagawa, E. Demler, and I. Bloch, Direct measurement of the Zak phase in topological Bloch bands, Nat. Phys. 9, 795 (2013).

[34] L. Lu, J. D. Joannopoulos, and M. Soljačić, Topological photonics, Nat. Photonics 8, 821 (2014).

[35] S. Mittal, S. Ganeshan, J. Fan, A. Vaez, and M. Hafezi, Measurement of topological invariants in a 2D photonic system, Nat. Photonics 10, 180 (2016).

[36] Q. Lin, M. Xiao, L. Yuan, and S. Fan, Photonic Weyl point in a two-dimensional resonator lattice with a synthetic frequency dimension, Nat. Commun. 7, 13731 (2016).

[37] M. Hafezi, E. A. Demler, M. D. Lukin, and J. M. Taylor, Robust optical delay lines with topological protection, Nat. Phys. 7, 907 (2011).

[38] M. Aidelsburger, M. Atala, M. Lohse, J. T. Barreiro, B. Paredes, and I. Bloch, Realization of the Hofstadter Hamiltonian with Ultracold Atoms in Optical Lattices, Phys. Rev. Lett. 111, 185301 (2013).

[39] N. Goldman, J. C. Budich, and P. Zoller, Topological quantum matter with ultracold gases in optical lattices, Nat. Phys. 12, 639 (2016).

[40] T. Ozawa, H. M. Price, A. Amo, N. Goldman, M. Hafezi, L. Lu, M. Rechtsman, D. Schuster, J. Simon, O. Zilberberg, and I. Carusotto, Topological photonics, arXiv:1802.04173.

[41] N. R. Cooper, J. Dalibard, and I. B. Spielman, Topological bands for ultracold atoms, arXiv:1803.00249.

[42] C. M. Bender and S. Boettcher, Real Spectra in Non-Hermitian Hamiltonians Having $\mathcal{P} \mathcal{T}$ Symmetry, Phys. Rev. Lett. 80, 5243 (1998).

[43] A. Mostafazadeh, Pseudo-Hermiticity versus PT symmetry: The necessary condition for the reality of the spectrum of a non-Hermitian Hamiltonian, J. Math. Phys. 43, 205 (2002).

[44] C. M. Bender, Making sense of non-Hermitian Hamiltonians, Rep. Prog. Phys. 70, 947 (2007). 
[45] K. G. Makris, R. El-Ganainy, D. N. Christodoulides, and Z. H. Musslimani, Beam Dynamics in $\mathcal{P} \mathcal{T}$ Symmetric Optical Lattices, Phys. Rev. Lett. 100, 103904 (2008).

[46] S. Klaiman, U. Günther, and N. Moiseyev, Visualization of Branch Points in $\mathcal{P} \mathcal{T}$-Symmetric Waveguides, Phys. Rev. Lett. 101, 080402 (2008).

[47] Z. H. Musslimani, K. G. Makris, R. El-Ganainy, and D. N. Christodoulides, Optical Solitons in $\mathcal{P} \mathcal{T}$ Periodic Potentials, Phys. Rev. Lett. 100, 030402 (2008).

[48] I. Rotter, A non-Hermitian Hamilton operator and the physics of open quantum systems, J. Phys. A 42, 153001 (2009).

[49] A. Guo, G. J. Salamo, D. Duchesne, R. Morandotti, M. Volatier-Ravat, V. Aimez, G. A. Siviloglou, and D. N. Christodoulides, $\mathcal{P} \mathcal{T}$-Symmetry Breaking and LaserAbsorber Modes in Optical Scattering Systems, Phys. Rev. Lett. 103, 093902 (2009).

[50] C. E. Rüter, K. G. Makris, R. El-Ganainy, D. N. Christodoulides, M. Segev, and D. Kip, Observation of paritytime symmetry in optics, Nat. Phys. 6, 192 (2010).

[51] Y. D. Chong, L. Ge, H. Cao, and A. D. Stone, Coherent Perfect Absorbers: Time-Reversed Lasers, Phys. Rev. Lett. 105, 053901 (2010).

[52] N. Moiseyev, Non-Hermitian Quantum Mechanics (Cambridge University Press, Cambridge, UK, 2011).

[53] M. Liertzer, L. Ge, A. Cerjan, A. D. Stone, H. E. Türeci, and S. Rotter, Pump-Induced Exceptional Points in Lasers, Phys. Rev. Lett. 108, 173901 (2012).

[54] A. Regensburger, M.-A. Miri, C. Bersch, J. Näger, G. Onishchukov, D. N. Christodoulides, and U. Peschel, Observation of Defect States in $\mathcal{P} \mathcal{T}$-Symmetric Optical Lattices, Phys. Rev. Lett. 110, 223902 (2013).

[55] B. Peng, S. K. Ozdemir, F. Lei, F. Monifi, M. Gianfreda, G. L. Long, S. Fan, F. Nori, C. M. Bender, and L. Yang, Parity-time-symmetric whispering-gallery microcavities, Nat. Phys. 10, 394 (2014).

[56] L. Chang, X. Jiang, S. Hua, C. Yang, J. Wen, L. Jiang, G. Li, G. Wang, and M. Xiao, Parity-time symmetry and variable optical isolation in active-passive-coupled microresonators, Nat. Photonics 8, 524 (2014).

[57] L. Feng, Z. J. Wong, R.-M. Ma, Y. Wang, and X. Zhang, Single-mode laser by parity-time symmetry breaking, Science 346, 972 (2014).

[58] H. Hodaei, M.-A. Miri, M. Heinrich, D. N. Christodoulides, and M. Khajavikhan, Parity-time-symmetric microring lasers, Science 346, 975 (2014).

[59] R. Fleury, D. Sounas, and A. Alù, An invisible acoustic sensor based on parity-time symmetry, Nat. Commun. 6, 5905 (2015).

[60] H. Cao and J. Wiersig, Dielectric microcavities: Model systems for wave chaos and non-Hermitian physics, Rev. Mod. Phys. 87, 61 (2015).

[61] F. Monticone, C. A. Valagiannopoulos, and A. Alù, ParityTime Symmetric Nonlocal Metasurfaces: All-Angle Negative Refraction and Volumetric Imaging, Phys. Rev. X 6, 041018 (2016).

[62] K. Ding, G. Ma, M. Xiao, Z. Q. Zhang, and C. T. Chan, Emergence, Coalescence, and Topological Properties of Multiple Exceptional Points and Their Experimental Realization, Phys. Rev. X 6, 021007 (2016).

[63] A. Cerjan, A. Raman, and S. Fan, Exceptional Contours and Band Structure Design in Parity-Time Symmetric
Photonic Crystals, Phys. Rev. Lett. 116, 203902 (2016).

[64] Z. Zhang, Y. Zhang, J. Sheng, L. Yang, M.-A. Miri, D. N. Christodoulides, B. He, Y. Zhang, and M. Xiao, Observation of Parity-Time Symmetry in Optically Induced Atomic Lattices, Phys. Rev. Lett. 117, 123601 (2016).

[65] L. Ge, Symmetry-protected zero-mode laser with a tunable spatial profile, Phys. Rev. A 95, 023812 (2017).

[66] W. Chen, S. K. Ozdemir, G. Zhao, J. Wiersig, and L. Yang, Exceptional points enhance sensing in an optical microcavity, Nature (London) 548, 192 (2017).

[67] H. Hodaei, A. U. Hassan, S. Wittek, H. Garcia-Gracia, R. El-Ganainy, D. N. Christodoulides, and M. Khajavikhan, Enhanced sensitivity at higher-order exceptional points, Nature (London) 548, 187 (2017).

[68] K. Kawabata, Y. Ashida, and M. Ueda, Information Retrieval and Criticality in Parity-Time-Symmetric Systems, Phys. Rev. Lett. 119, 190401 (2017).

[69] Y. Ashida, S. Furukawa, and M. Ueda, Parity-time-symmetric quantum critical phenomena, Nat. Commun. 8, 15791 (2017).

[70] S. Assawaworrarit, X. Yu, and S. Fan, Robust wireless power transfer using a nonlinear parity-time-symmetric circuit, Nature (London) 546, 387 (2017).

[71] H. Zhou, C. Peng, Y. Yoon, C. W. Hsu, K. A. Nelson, L. Fu, J. D. Joannopoulos, M. Soljačić, and B. Zhen, Observation of bulk Fermi arc and polarization half charge from paired exceptional points, Science 359, 1009 (2018).

[72] G. Harari, M. A. Bandres, Y. Lumer, M. C. Rechtsman, Y. D. Chong, M. Khajavikhan, D. N. Christodoulides, and M. Segev, Topological insulator laser: Theory, Science 359, eaar4003 (2018).

[73] M. A. Bandres, S. Wittek, G. Harari, M. Parto, J. Ren, M. Segev, D. Christodoulides, and M. Khajavikhan, Topological insulator laser: Experiments, Science 359, eaar4005 (2018).

[74] L. Feng, R. El-Ganainy, and L. Ge, Non-Hermitian photonics based on parity-time symmetry, Nat. Photonics 11, 752 (2017).

[75] R. El-Ganainy, K. G. Makris, M. Khajavikhan, Z. H. Musslimani, S. Rotter, and D. N. Christodoulides, Non-Hermitian physics and PT symmetry, Nat. Phys. 14, 11 (2018).

[76] S. Diehl, E. Rico, M. A. Baranov, and P. Zoller, Topology by dissipation in atomic quantum wires, Nat. Phys. 7, 971 (2011).

[77] Y. C. Hu and T. L. Hughes, Absence of topological insulator phases in non-Hermitian $\mathcal{P} \mathcal{T}$-symmetric Hamiltonians, Phys. Rev. B 84, 153101 (2011).

[78] K. Esaki, M. Sato, K. Hasebe, and M. Kohmoto, Edge states and topological phases in non-Hermitian systems, Phys. Rev. B 84, 205128 (2011).

[79] G. Q. Liang and Y. D. Chong, Optical Resonator Analog of a Two-Dimensional Topological Insulator, Phys. Rev. Lett. 110, 203904 (2013).

[80] B. Zhu, R. Lü, and S. Chen, $\mathcal{P} \mathcal{T}$ symmetry in the nonHermitian Su-Schrieffer-Heeger model with complex boundary potentials, Phys. Rev. A 89, 062102 (2014).

[81] C. Yuce, Topological phase in a non-Hermitian $\mathcal{P} \mathcal{T}$ symmetric system, Phys. Lett. A 379, 1213 (2015).

[82] X. Wang, T. Liu, Y. Xiong, and P. Tong, Spontaneous $\mathcal{P} \mathcal{T}$-symmetry breaking in non-Hermitian Kitaev 
and extended Kitaev models, Phys. Rev. A 92, 012116 (2015).

[83] H. Zhao, S. Longhi, and L. Feng, Robust light state by quantum phase transition in non-Hermitian optical materials, Sci. Rep. 5, 17022 (2015).

[84] S. Malzard, C. Poli, and H. Schomerus, Topologically Protected Defect States in Open Photonic Systems with Non-Hermitian Charge-Conjugation and Parity-Time Symmetry, Phys. Rev. Lett. 115, 200402 (2015).

[85] M. S. Rudner and L. S. Levitov, Topological Transition in a Non-Hermitian Quantum Walk, Phys. Rev. Lett. 102, 065703 (2009).

[86] H. Schomerus, Topologically protected midgap states in complex photonic lattices, Opt. Lett. 38, 1912 (2013).

[87] C. Poli, M. Bellec, U. Kuhl, F. Mortessagne, and H. Schomerus, Selective enhancement of topologically induced interface states in a dielectric resonator chain, Nat. Commun. 6, 6710 (2015).

[88] J. M. Zeuner, M. C. Rechtsman, Y. Plotnik, Y. Lumer, S. Nolte, M. S. Rudner, M. Segev, and A. Szameit, Observation of a Topological Transition in the Bulk of a NonHermitian System, Phys. Rev. Lett. 115, 040402 (2015).

[89] S. Weimann, M. Kremer, Y. Plotnik, Y. Lumer, S. Nolte, K. G. Makris, M. Segev, M. C. Rechtsman, and A. Szameit, Topologically protected bound states in photonic parity-timesymmetric crystals, Nat. Mater. 16, 433 (2017).

[90] H. Zhao, P. Miao, M. H. Teimourpour, S. Malzard, R. El-Ganainy, H. Schomerus, and L. Feng, Topological hybrid silicon microlasers, Nat. Commun. 9, 981 (2018).

[91] M. Pan, H. Zhao, P. Miao, S. Longhi, and L. Feng, Photonic zero mode in a non-Hermitian photonic lattice, Nat. Commun. 9, 1308 (2018).

[92] M. Parto, S. Wittek, H. Hodaei, G. Harari, M. A. Bandres, J. Ren, M. C. Rechtsman, M. Segev, D. N. Christodoulides, and M. Khajavikhan, Edge-Mode Lasing in 1D Topological Active Arrays, Phys. Rev. Lett. 120, 113901 (2018).

[93] S. Lieu, Topological phases in the non-Hermitian SuSchrieffer-Heeger model, Phys. Rev. B 97, 045106 (2018).

[94] R. Wang, X. Z. Zhang, and Z. Song, Dynamical topological invariant for non-Hermitian Rice-Mele model, Phys. Rev. A 98, 042120 (2018).

[95] K. Kawabata, Y. Ashida, H. Katsura, and M. Ueda, Paritytime-symmetric topological superconductor, Phys. Rev. B 98, 085116 (2018).

[96] K. Kawabata, S. Higashikawa, Z. Gong, Y. Ashida, and M. Ueda, Topological unification of time-reversal and particlehole symmetries in non-Hermitian physics, Nat. Commun. 10, 297 (2019).

[97] L. J. Lang, Y. Wang, H. Wang, and Y. D. Chong, Effects of non-hermiticity on su-schrieer-heeger defect states, Phys. Rev. B 98, 094307 (2018).

[98] C. A. Downing and G. Weick, Topological collective plasmons in bipartite chains of metallic nanoparticles, Phys. Rev. B 95, 125426 (2017).

[99] L. Jin, P. Wang, and Z. Song, Su-Schrieffer-Heeger chain with one pair of $\mathcal{P} \mathcal{T}$-symmetric defects, Sci. Rep. 7, 5903 (2017).

[100] M. Klett, H. Cartarius, D. Dast, J. Main, and G. Wunner, Relation between $\mathcal{P} \mathcal{T}$-symmetry breaking and topologically nontrivial phases in the Su-Schrieffer-Heeger and Kitaev models, Phys. Rev. A 95, 053626 (2017).
[101] H. Menke and M. M. Hirschmann, Topological quantum wires with balanced gain and loss, Phys. Rev. B 95, 174506 (2017).

[102] L. Jin, Topological phases and edge states in a non-Hermitian trimerized optical lattice, Phys. Rev. A 96, 032103 (2017).

[103] W. Hu, H. Wang, P. P. Shum, and Y. D. Chong, Exceptional points in a non-Hermitian topological pump, Phys. Rev. B 95, 184306 (2017).

[104] J. González and R. A. Molina, Topological protection from exceptional points in Weyl and nodal-line semimetals, Phys. Rev. B 96, 045437 (2017).

[105] L. Xiao, X. Zhan, Z. H. Bian, K. K. Wang, X. Zhang, X. P. Wang, J. Li, K. Mochizuki, D. Kim, N. Kawakami, W. Yi, H. Obuse, B. C. Sanders, and P. Xue, Observation of topological edge states in parity-time-symmetric quantum walks, Nat. Phys. 13, 1117 (2017).

[106] W. Zhu, X. Fang, D. Li, Y. Sun, Y. Li, Y. Jing, and H. Chen, Simultaneous Observation of a Topological Edge State and Exceptional Point in an Open and Non-Hermitian Acoustic System, Phys. Rev. Lett. 121, 124501 (2018).

[107] D. Leykam, K. Y. Bliokh, C. Huang, Y. D. Chong, and F. Nori, Edge Modes, Degeneracies, and Topological Numbers in Non-Hermitian Systems, Phys. Rev. Lett. 118, 040401 (2017).

[108] Y. Xu, S.-T. Wang, and L.-M. Duan, Weyl Exceptional Rings in a Three-Dimensional Dissipative Cold Atomic Gas, Phys. Rev. Lett. 118, 045701 (2017).

[109] H. Shen, B. Zhen, and L. Fu, Topological Band Theory for Non-Hermitian Hamiltonians, Phys. Rev. Lett. 120, 146402 (2018).

[110] A. Y. Song, P. B. Catrysse, and S. Fan, Broadband Control of Topological Nodes in Electromagnetic Fields, Phys. Rev. Lett. 120, 193903 (2018).

[111] C. Yuce, Edge states at the interface of non-Hermitian systems, Phys. Rev. A 97, 042118 (2018).

[112] C. Yin, H. Jiang, L. Li, R. Lü, and S. Chen, Geometrical meaning of winding number and its characterization of topological phases in one-dimensional chiral non-Hermitian systems, Phys. Rev. A 97, 052115 (2018).

[113] H. Shen and L. Fu, Quantum Oscillation from In-Gap States and a Non-Hermitian Landau Level Problem, Phys. Rev. Lett. 121, 026403 (2018).

[114] S. Lieu, Topological symmetry classes for non-Hermitian models and connections to the bosonic Bogoliubov-de Gennes equation, Phys. Rev. B 98, 115135 (2018).

[115] H. Jiang, C. Yang, and S. Chen, Topological invariants, phase diagrams and discrepancy for non-Hermitian systems without chiral symmetry, Phys. Rev. A 98, 052116 (2018).

[116] S. Malzard and H. Schomerus, Bulk and edge-state arcs in non-Hermitian coupled-resonator arrays, Phys. Rev. A 98, 033807 (2018).

[117] M. Klett, H. Cartarius, D. Dast, J. Main, and G. Wunner, Topological edge states in the Su-Schrieer-Heeger model subject to balanced particle gain and loss, arXiv:1802.06128.

[118] In this work, the term "bulk-boundary correspondence" refers to the conventional bulk-boundary correspondence.

[119] V. M. Martinez Alvarez, J. E. Barrios Vargas, and L. E. F. Foa Torres, Non-Hermitian robust edge states in one dimension: Anomalous localization and eigenspace condensation at exceptional points, Phys. Rev. B 97, 121401(R) (2018).

[120] T. E. Lee, Anomalous Edge State in a Non-Hermitian Lattice, Phys. Rev. Lett. 116, 133903 (2016). 
[121] Y. Xiong, Why does bulk boundary correspondence fail in some non-Hermitian topological models, J. Phys. Commun. 2, 035043 (2018).

[122] F. K. Kunst, E. Edvardsson, J. C. Budich, and E. J. Bergholtz, Biorthogonal Bulk-Boundary Correspondence in Non-Hermitian Systems, Phys. Rev. Lett. 121, 026808 (2018).

[123] S. Yao and Z. Wang, Edge States and Topological Invariants of Non-Hermitian Systems, Phys. Rev. Lett. 121, 086803 (2018).

[124] Z. Gong, Y. Ashida, K. Kawabata, K. Takasan, S. Higashikawa, and M. Ueda, Topological Phases of Non-Hermitian Systems, Phys. Rev. X 8, 031079 (2018).

[125] C. H. Lee and R. Thomale, Anatomy of skin modes and topology in non-Hermitian systems, arXiv:1809.02125.

[126] Z. Yang and J. Hu, Nodal line semimetals under nonHermitian perturbations-Emerging Hopf-link exceptional line semimetals, arXiv:1807.05661.

[127] H. Wang, J. Ruan, and H. Zhang, Non-Hermitian nodal-line semimetals, arXiv:1808.06162.

[128] V. M. Martinez Alvarez, J. E. Barrios Vargas, M. Berdakin, and L. E. F. Foa Torres, Topological states of non-Hermitian systems, Eur. Phys. J. Spec. Top. 227, 1295 (2018).

[129] S. Yao, F. Song, and Z. Wang, Non-Hermitian Chern Bands, Phys. Rev. Lett. 121, 136802 (2018).

[130] K. Kawabata, K. Shiozaki, and M. Ueda, Anomalous helical edge states in a non-Hermitian Chern insulator, Phys. Rev. B 98, 165148 (2018).

[131] W. P. Su, J. R. Schrieffer, and A. J. Heeger, Solitons in Polyacetylene, Phys. Rev. Lett. 42, 1698 (1979).

[132] N. Hatano and D. R. Nelson, Localization Transitions in Non-Hermitian Quantum Mechanics, Phys. Rev. Lett. 77, 570 (1996).

[133] S. Longhi, D. Gatti, and G. Della Valle, Non-Hermitian transparency and one-way transport in low-dimensional lattices by an imaginary gauge field, Phys. Rev. B 92, 094204 (2015).

[134] B. Midya, H. Zhao, and L. Feng, Non-Hermitian photonics promises exceptional topology of light, Nat. Commun. 9, 2674 (2018).

[135] See Supplemental Material at http://link.aps.org/ supplemental/10.1103/PhysRevB.99.081103 for more details on the imaginary gauge field and non-Hermitian $\mathrm{AB}$ effect, the bulk state inverse participation ratio, the proof of identical energy spectra of systems $a$ and $b$ under OBC, the chiral inversion symmetric systems, the topological characterization, the finite effect on energy spectra, the edge states for a system with a defective unit cell, the equivalence between non-Hermitian SSH models and Creutz ladders, and the 2D non-Hermitian topological systems.

[136] X. Z. Zhang and Z. Song, Momentum-independent reflectionless transmission in the non-Hermitian time-reversal symmetric system, Ann. Phys. (NY) 339, 109 (2013).

[137] K. Fang, Z. Yu, and S. Fan, Photonic Aharonov-Bohm Effect Based on Dynamic Modulation, Phys. Rev. Lett. 108, 153901 (2012).

[138] K. Fang, Z. Yu, and S. Fan, Realizing effective magnetic field for photons by controlling the phase of dynamic modulation, Nat. Photonics 6, 782 (2012).

[139] E. Li, B. J. Eggleton, K. Fang, and S. Fan, Photonic AharonovBohm effect in photon-phonon interactions, Nat. Commun. 5, 3225 (2014).
[140] M. Hafezi, Measuring Topological Invariants in Photonic Systems, Phys. Rev. Lett. 112, 210405 (2014).

[141] L. Jin and Z. Song, Parity-time symmetry under magnetic flux, Phys. Rev. A 93, 062110 (2016).

[142] L. Jin, P. Wang, and Z. Song, One-way light transport controlled by synthetic magnetic fluxes and $\mathcal{P} \mathcal{T}$-symmetric resonators, New J. Phys. 19, 015010 (2017).

[143] L. Jin, Parity-time-symmetric coupled asymmetric dimers, Phys. Rev. A 97, 012121 (2018).

[144] Peierls phase factor refers to $e^{ \pm i \Phi}$ [39], where $\Phi$ is imaginary $\Phi=i \phi$ for the asymmetric coupling $\sqrt{\mu \nu} e^{\mp \phi}$

[145] W. D. Heiss and H. L. Harney, The chirality of exceptional points, Eur. Phys. J. D 17, 149 (2001).

[146] C. Dembowski, H.-D. Gräf, H. L. Harney, A. Heine, W. D. Heiss, H. Rehfeld, and A. Richter, Experimental Observation of the Topological Structure of Exceptional Points, Phys. Rev. Lett. 86, 787 (2001).

[147] M. V. Berry, Physics of Nonhermitian Degeneracies, Czech. J. Phys. 54, 1039 (2004).

[148] A. A. Mailybaev, O. N. Kirillov, and A. P. Seyranian, Geometric phase around exceptional points, Phys. Rev. A 72, 014104 (2005).

[149] M. Müller and I. Rotter, Exceptional points in open quantum systems, J. Phys. A: Math. Theor. 41, 244018 (2008).

[150] R. Uzdin, A. Mailybaev, and N. Moiseyev, On the observability and asymmetry of adiabatic state flips generated by exceptional points, J. Phys. A 44, 435302 (2011).

[151] W. D. Heiss, The physics of exceptional points, J. Phys. A 45, 444016 (2012).

[152] S.-D. Liang and G.-Y. Huang, Topological invariance and global Berry phase in non-Hermitian systems, Phys. Rev. A 87, 012118 (2013).

[153] B. Zhen, C. W. Hsu, Y. Igarashi, L. Lu, I. Kaminer, A. Pick, S.-L. Chua, J. D. Joannopoulos, and M. Soljačić, Spawning rings of exceptional points out of Dirac cones, Nature (London) 525, 354 (2015).

[154] J. Doppler, A. A. Mailybaev, J. Böhm, U. Kuhl, A. Girschik, F. Libisch, T. J. Milburn, P. Rabl, N. Moiseyev, and S. Rotter, Dynamically encircling an exceptional point for asymmetric mode switching, Nature (London) 537, 76 (2016).

[155] H. Xu, D. Mason, L. Jiang, and J. G. E. Harris, Topological energy transfer in an optomechanical system with exceptional points, Nature (London) 537, 80 (2016).

[156] D. Heiss, Circling exceptional points, Nat. Phys. 12, 823 (2016).

[157] A. U. Hassan, B. Zhen, M. Soljačić, M. Khajavikhan, and D. N. Christodoulides, Dynamically Encircling Exceptional Points: Exact Evolution and Polarization State Conversion, Phys. Rev. Lett. 118, 093002 (2017).

[158] T. Goldzak, A. A. Mailybaev, and N. Moiseyev, Light Stops at Exceptional Points, Phys. Rev. Lett. 120, 013901 (2018).

[159] X.-L. Zhang, S. Wang, B. Hou, and C. T. Chan, Dynamically Encircling Exceptional Points: In situ Control of Encircling Loops and the Role of the Starting Point, Phys. Rev. X 8, 021066 (2018).

[160] K. Ding, G. Ma, Z. Q. Zhang, and C. T. Chan, Experimental Demonstration of an Anisotropic Exceptional Point, Phys. Rev. Lett. 121, 085702 (2018). 
[161] L. Jin and Z. Song, Incident Direction Independent Wave Propagation and Unidirectional Lasing, Phys. Rev. Lett. 121, 073901 (2018).

[162] Biorthogonal norms of the eigenstates are unaffected under the imaginary gauge field (see Supplemental Material A [135]).

[163] L. Ge, Anomalous minimum and scaling behavior of localization length near an isolated flat band, Ann. Phys. (Berlin) 529, 1600182 (2017).

[164] For system $b, \mathcal{S P}=\sigma_{x} \otimes \sigma_{y}$ and $U_{\mathcal{S P}}=P_{2 n} \otimes \sigma_{y}$ in Eq. (2), where $\otimes$ is the Kronecker product, and $P_{2 n}$ is a $90^{\circ}$ rotation of the $2 n \times 2 n$ identical matrix $I_{2 n}$.

[165] K. Takata and M. Notomi, Photonic Topological Insulating Phase Induced Solely by Gain and Loss, Phys. Rev. Lett. 121, 213902 (2018).
[166] K. Sun, W. V. Liu, A. Hemmerich, and S. Das Sarma, Topological semimetal in a fermionic optical lattice, Nat. Phys. 8, 67 (2012).

[167] J. M. Hou, Hidden-Symmetry-Protected Topological Semimetals on a Square Lattice, Phys. Rev. Lett. 111, 130403 (2013).

[168] If the asymmetric intracell coupling $\mu-v$ at system boundary vanishes instead of the intercell coupling $t_{2}$, the conclusion for the existence of zero edge states is inverse as in the regions $t_{1}^{2}<\gamma^{2}-t_{2}^{2}$ and $t_{1}^{2}>\gamma^{2}+t_{2}^{2}$.

[169] M. Creutz, End States, Ladder Compounds, and Domain-Wall Fermions, Phys. Rev. Lett. 83, 2636 (1999). 